\title{
INFLUENCE OF CORRELATED THERMAL VIBRATIONS ON TEMPERATURE DEPENDENCE OF SURFACE PEAK
}

\author{
T. Gwizdalla and J. Czerbniak
}

Dept. of Solid State Physics, University of Lódź

Pomorska 149/153, 90-236 Lódż, Poland

(Received May 9, 1995; revised version September 22, 1995)

The influence of correlation between thermal displacements of atoms in string on the calculated Rutherford backscattering spectra under channelling conditions was studied. This correlation was incorporated into the existing Monte Carlo computer code by means of sampling the displacements from distribution transformed by the variance-covariance matrix. Various methods of implementing the correlation into calculation were used. As a result the deformation of energy spectrum was obtained. The explicit decrease in surface peak due to the correlation was observed. The significance of this effect decreases with increasing temperature. Calculation were performed in a wide region of temperatures, up to the melting point.

PACS numbers: $61.80 . \mathrm{Mk}, 63.20 .-\mathrm{e}$

\section{Introduction}

There is a strong interest in taking into account the effect of correlations between thermal displacements of crystal atoms by studying the ion penetration in solid. This effect may cause changes in various characteristics, observed by using medium energy ion scattering (MEIS) technique, especially for high symmetry directions (e.g. $\langle 100\rangle,\langle 110\rangle)$, where the correlation coefficients are expected to be especially significant. Many Monte Carlo computer codes, created to analysis of ion motion in solids are based on theoretical models which assume the negligibility of such correlations [1-3]. They are also not included in calculations, devoted to the study of melting [4]. In some recent papers, there were presented results obtained with correlation included. These are, however, not consistent one to another. Alliney et al. [5] showed that in the case of blocking, the correlation length is too short compared to the string length needed to generate the blocking pattern, therefore the shapes of blocking dips are very similar. On the other hand, Barrett and Jackson [6] demonstrated that some quantities concerning the channelling (surface 
peak, minimum yield) are significantly (of the magnitude 10-15\%) changed, when taking the correlations into consideration. Differences may probably be caused by various methods of sampling of atomic displacements. Although both methods based on the transforming of variance-covariance matrix by the Cholesky factorization, the Barrett-Jackson method assumes calculating of positions of further atoms using the special "shifting" algorithm.

\section{Calculation procedure}

In the calculations we used our own program which is based on the following assumptions: atomic collisions are considered within the frame of binary collision model using the screened Coulomb potential with Moliere screening function [7]; electronic energy losses are taken from formula proposed by Dettman and Robinson [8] which includes separately energy transfer to lower shell electrons and valence electrons; for individual collision the nuclear encounter probability is calculated. In this way we may simply obtain the magnitude of a surface peak, expressed in number of visible atoms per row, adding the probabilities of collisions with successive atoms.

Standard model of calculation assumes sampling of atomic displacements from Gaussian distribution around equilibrium positions. Obviously, in this model the correlation coefficients between the position of atoms are equal to zero. Presenting the components of displacements of all $n$ considered atoms in the row in the direction perpendicular to the row in the form of $n$-dimensional vector

$$
\boldsymbol{x}=\left[\begin{array}{c}
x_{1} \\
x_{2} \\
x_{3} \\
\vdots \\
x_{n}
\end{array}\right],
$$

we may write the probability density function of $n$-dimensional normal distribution

$$
f(x)=\frac{1}{\sqrt{(2 \pi)^{n}\left|M^{-1}\right|}} \exp \left(-1 \text { rver } 2 x^{\mathrm{T}} M^{-1} x\right)
$$

where $\boldsymbol{M}$ is the variance-covariance matrix. In the case described above $\boldsymbol{M}=\boldsymbol{I} \sigma^{2}$; $I$ - identity matrix, $\sigma^{2}$ - root mean square amplitude of vibration, which may be obtained from the phonon theory.

The values of correlated thermal displacements should have the probability density function described by the formula (2), with nonzero elements out of diagonal. These elements are related to the equal-time correlation coefficients, defined as

$$
M_{i j}=\frac{\left\langle x_{i}(t) x_{j}(t)\right\rangle}{\left\langle x_{i}(t) x_{i}(t)\right\rangle} .
$$

These values were taken from the paper of Nelson et al. [9]. We will not take into account non-equal-time correlations because the distance covered by the particle during one period of vibration is very long, as compared with lattice constant for 
energies considered in this paper. The high tempcrature $(T>\Theta$, where $\Theta-$ Debye temperature) approximation assumes that they have very simple dependence on distance between considered atoms

$$
M_{i j}=\left(2 \eta^{*} n\right)^{-1} \text {. }
$$

In this formula $n$ denotes the distance between atoms expressed in units of distance to the nearest neighbour and $\eta=(6 \sqrt{2} / \pi)^{1 / 3}$ comes from the determination of the "Debye sphere" radius.

The values of correlation coefficients obtained for two directions in fcc crystal are listed in Table.

\section{TABLE}

The values of the correlation coefficients for $\langle 100\rangle$ and $\langle 110\rangle$ directions in fcc crystal.

\begin{tabular}{c|c|c|c|c|c|c|c}
\hline \hline & $i$ & 1 & 2 & 3 & 4 & 5 & 6 \\
\hline$\langle 100\rangle$ & $\beta_{i}$ & 0.25 & 0.13 & 0.08 & 0.06 & 0.05 & 0.04 \\
$\langle 110\rangle$ & $\beta_{i}$ & 0.36 & 0.18 & 0.12 & 0.09 & 0.07 & 0.06
\end{tabular}

Factorizing matrix $M: M=L L^{\mathrm{T}}$, and defining $\boldsymbol{x}^{\prime}=\boldsymbol{L}^{-1} \boldsymbol{x}$, one can rewrite (2) in terms of $x^{\prime}$ :

$$
f\left(x^{\prime}\right)=\frac{1}{\sqrt{(2 \pi)^{n}\left|M^{-1}\right|}} \exp \left(-\frac{1}{2} x^{\prime \mathrm{T}^{\prime}} x^{\prime}\right) .
$$

One notices that every component $\boldsymbol{x}_{i}^{\prime}$ has $N(0,1)$ distribution.

Since the vector $x^{\prime}$ contains $n$ components, whose values are sampled from normal distribution, one can easily obtain correlated displacements $x$ transforming

$$
\boldsymbol{x}=\boldsymbol{L} \boldsymbol{x}^{\prime} \text {. }
$$

The correlations were taken up to sixth neighbour and incorporated in two described below methods, in order to make possible the comparison of their results:

a) the perpendicular components of positions of atoms in row are calculated for the successive groups separately (if we denote the atoms with their numbers in the string we may schematically present this method as: $[1,2,3,4,5,6]$ $[7,8,9,10,11,12] \ldots)$;

b) after first calculation of positions we treat three last values as sampled from Gaussian distribution i.e. as the first values of a new $\boldsymbol{x}^{\prime}$ vector (in the notation described in previous point $[1,2,3,4,5,6][4,5,6,7,8,9][7,8,9,10,11,12])$.

The Nelson, Thomson and Montgomery formula (4) is based on some simplifications. The Brillouin zone was replaced by a sphere of equal volume in reciprocal space and the symmetric distribution of the wave vectors of created and annihilated phonons was assumed. Especially the second condition may not be valid for the surface, therefore one has to underline that these listed earlier values describe correctly only the bulk conditions. 
In order to include surface effects we should take into account in particular two phenomena:

1) relaxation of lattice parameter,

2 ) increase in the mean square amplitude of thermal vibrations.

The first one is omitted in our calculation. We think that the displacement of an atom parallel to the crystallographic direction, which is the direction of motion of an ion, does not cause the significant effect on the shape of shadow cone, if compared to the perpendicular one. Because of its magnitude the second effect cannot be neglected. The preliminary results of our group [12] obtained on the basis of the model of lattice dynamics in harmonic approximation adapted to the surface atoms have shown that for special type of surfaces the amplitude of their vibrations may be up to $50 \%$ greater than that in the bulk.

\section{Results}

In order to compare various methods of approaching the correlation effect the calculations were performed in three ways: without correlation, using simple Cholesky method, using Barrett-Jackson's algorithm.

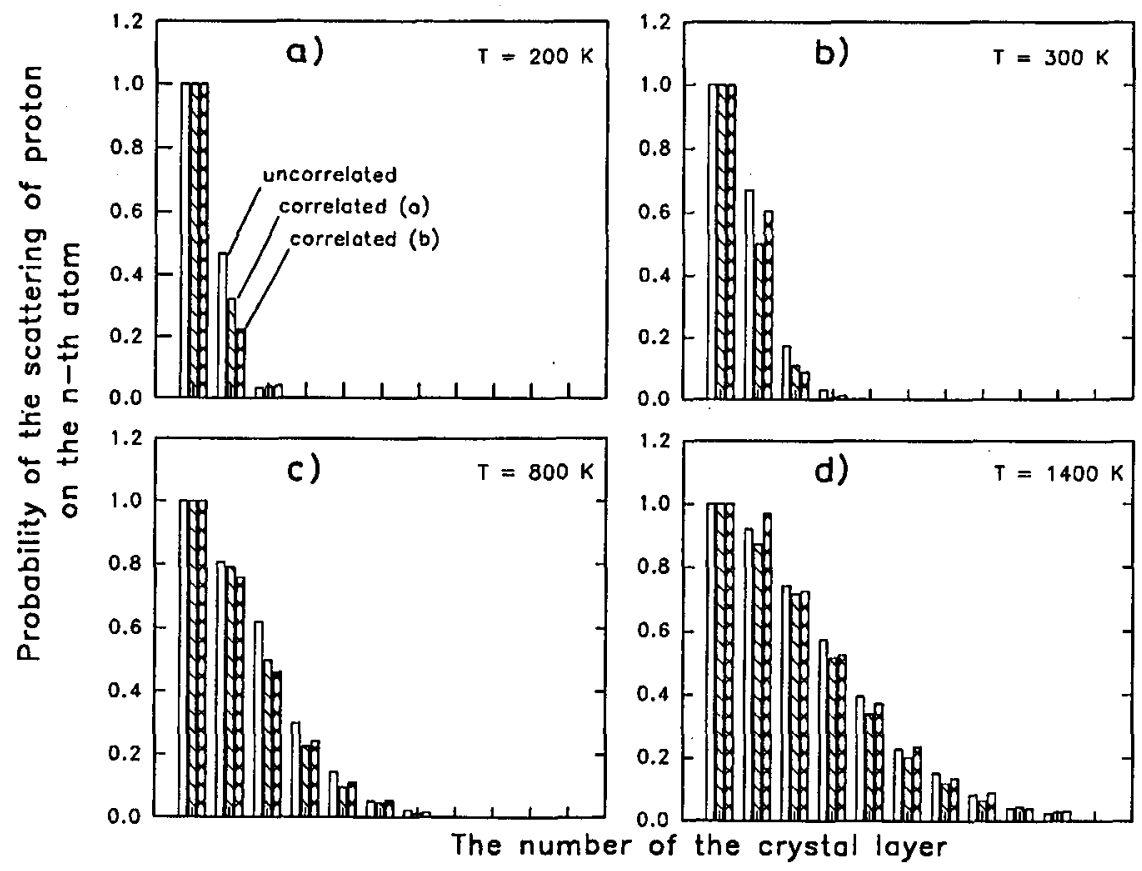

Fig. 1. Temperature dependence of the probability of scattering of $100 \mathrm{keV}$ protons on successive atoms along the $\langle 100\rangle$ row of the silicon single crystal for various methods of including the correlation effect. Plots: (a) $-200 \mathrm{~K},(\mathrm{~b})-300 \mathrm{~K}$, (c) $-800 \mathrm{~K}$, (d) $1400 \mathrm{~K}$. 


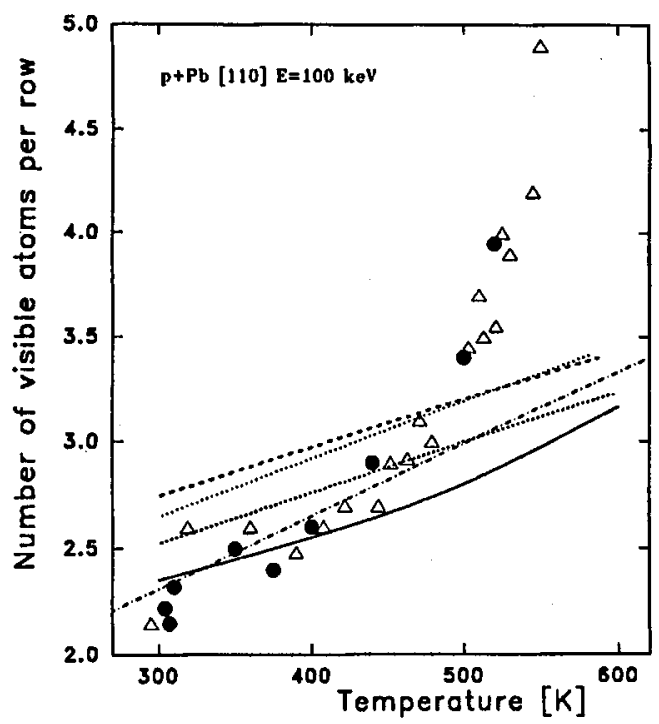

Fig. 2. Number of atoms visible by the beam of $100 \mathrm{keV}$ protons impinging perpendicularly the [110] surface of the $\mathrm{Pb}$ single crystal: long dashed line - equal amplitude, without correlation, short dashed line - equal amplitude, with correlation, dotted line - changed amplitude, without correlation, solid line - changed amplitude, with correlation, triangles - Ref. [10], dots - Ref. [11], dashed-dotted line - simulation from Ref. [11].

The first plot was made for silicon, which is characterised by a relatively high melting temperature $(1700 \mathrm{~K})$, therefore the obtained results can be presented in the wider temperature range. In Fig. 1 there are presented the probabilities of scattering of $100 \mathrm{keV}$ protons moving along the $\langle 001)$ axis in silicon by the successive crystal atoms, normalised to the first atom in row. The first bar corresponds to the calculation without correlation, the second one, described "correlated (a)" corresponds to the results obtained using only the factorization and the third one "correlated (b)" shows the influence of the use of Barrett-Jackson's method.

It may be seen that in the case of correlations included the hitting probability is smaller than for independent displacements. It can be however observed that for the temperatures much higher than room temperature, but still far from the melting point a significance of this effect becomes less important. Also the simple connection between results obtained for various procedures of incorporation the correlations cannot be determined unambiguously. The ratio of results obtained from Barrett-Jackson's procedure to those from simple sampling is for low temperatures less than 1, and explicitly increases with increase in temperature.

All further calculations were made for the protons moving along the $\langle 110\rangle$ direction in lead. The results for the various combinations of effects included into the code are presented in Fig. 2.

The formula "changed amplitude" corresponds to the calculations in which the amplitude of surface atom vibrations greater than in bulk was assumed. The 
values of enlargement factors are according to Ref. [12] taken to be: $n_{1}=1.6$, $n_{2}=1.3, n_{3}=1.05, n_{m}=1$ for $m>3$, where the index determines the layer number. This assumption changes only slightly our presented earlier equations. Matrix $M$ in (2) must be now written $M=I\left(\sigma^{2}\right)$, where $\left(\sigma^{2}\right)$ is the $n$-dimensional vector, whose elements $\left(\sigma^{2}\right)_{j}$ correspond to the square of vibrational amplitude of atoms in layer $\left(\sigma^{2}\right)_{j}=\left(n_{j}^{*} \sigma_{\mathrm{BULK}}\right)^{2}$.

The plot in Fig. 2 presents the temperature dependence of the number of visible atoms per row. If considered separately, the correlations and the increase in surface atom vibration amplitude produce only small changes in the shape of plotted functions. This is due to the fact that if atoms of first few layers vibrate with the greater amplitude, the successive atoms along the axis may be seldom covered by the preceding ones, and thus are "better seen" by the impinging particle. This causes that the line for this case lies higher than for standard calculation, and the difference between them seems to be constant, only the small tendency to decreasing this difference for big temperatures may be observed.

Also the results for correlation included are in good agreement with those, which can be expected after simple analysis. If the position of an atom is correlated with the position of the preceding one then it can be found with greater probability in the area of so-called shadow cone thus the collision will not take place and finally the total number of visible atoms will decrease. The absolute value of difference between yields for correlated and uncorrelated atom positions (long-dashed and short-dashed lines) does not change in the considered region of temperatures, and is equal to about 0.2 .

The fourth line, for common considering of correlations and increased surface atoms vibration amplitude lies considerably below three others. Such a result can be understood as a shadowing of quite all, other than belonging to the first surface layer atoms by the first one.

The experimental points present significant deviations from the unique line, which should describe the studied dependence. Those deviations are not well seen on the original plots in Refs. [10] and [11], where the vertical scale is much wider, as compared with showed here in order to present the surface peak value for the temperatures close to the melting temperature where it reaches very high values. In the quoted references there is shown that the critical temperature where the experimental points form characteristic curvature is approximately $450 \mathrm{~K}$. Above this point the positional disordering of surface and subsurface layers causes very fast increase in number of visible atoms. It should be mentioned that only the lowest line on the plot presents the similar curvature, however not so significant. Also the results of simulations presented in Ref. [11] (dashed-dotted line in Fig. 2) do not describe well this effect.

Comparing the calculated yield for the temperatures below the critical point it may be observed that among four presented lines three lie explicitly above the experimental data. Only the solid line, obtained using procedure with correlations, as well as the increase in vibrations amplitude in surface layers included may estimate well experimental values.

It should be also pointed out that the contaminations on the surface of crystal have a great influence on the experimentally observed value of surface 
peak. The contaminations create an amorphous layer on the top of the sample thus increasing obtained values. In order to compare the results of our calculations to the experiment made in some characteristic conditions we should assume the existence of such a layer having the thickness equal to the thickness determined in experiment. In the case of papers cited it was proven [12] that the surface was well cleaned and ordered and the amorphous layer must not be taken into account.

\section{Conclusion}

We made simulations of number of $100 \mathrm{keV}$ protons backscattered from $\langle 110\rangle$ surface of lead. We find that correlations are not negligible, when taking into consideration interaction of particle with several first layers of crystal. The inclusion of various effects related to the atomic vibrations into the simulation code influences significantly observed characteristics changing explicitly the shape of dependence of surface peak on the temperature.

\section{References}

[1] J.H. Barrett, Phys. Rev. B 3, 1527 (1971).

[2] P.J.M. Smulders, D.O. Boerma, Nucl. Instrum. Methods Phys. Res. B 29, 471 (1987).

[3] A. Dygo, A. Turos, Phys. Lett. A 127, 281 (1988).

[4] J.F. van der Veen, J.W.M. Frenken, Surf. Sci. 251/252, 1 (1991).

[5] S. Alliney, F. Malaguti, E. Verondini, Nucl. Instrum. Methods Phys. Res. B 28, 10 (1987).

[6] J.H. Barrett, D.P. Jackson, Nucl. Instrum. Methods Phys. Res. 170, 115 (1980);

J.H. Barrett, D.P. Jackson, Comp. Phys. Commun. 13, 157 (1977).

[7] G. Moliere, Z. Nat.forsch A 2, 133 (1947).

[8] K. Dettman, M.T. Robinson, Phys. Rev. B 10, 1 (1974).

[9] R.S. Nelson, M.W. Thompson, H. Montgomery, Philos. Mag. 1, 1385 (1962).

[10] J.W.M. Frenken, J.F. van der Veen, Phys. Rev. Lett. 54, 134 (1985).

[11] J.F. van der Veen, J.W.M. Frenken, Surf. Sci. 251, 1 (1991).

[12] J. Czerbniak, L. Wojtczak, L. Valenta, T. Gwizdałła, to be published. 